\title{
Tweaking Metabolic Networks: A Design Method
}

\author{
Surajit Panja $^{1} \cdot$ Sourav Patra $^{2} \cdot$ Anirban Mukherjee $^{2}$
}

Received: 24 April 2016/ Accepted: 6 May 2016/Published online: 25 May 2016

(C) Indian National Academy of Engineering 2016

\begin{abstract}
A robust synthesis technique is devised for synergism and saturation systems, commonly known as S-systems, for steering steady-states of the anaerobic fermentation pathway (AFP) of Saccharomyces cerevisae. The stability, steady-state error and robustness aspects are analyzed. The synthesis problem is framed in a computationally efficient convex optimization framework. The linear matrix inequalities are formulated for minimization of steady-state error, improvement of robustness and utilization of minimum control inputs ensuring the stability of the synthetic AFP.
\end{abstract}

Keywords Metabolic network $\cdot$ Robustness $\cdot$ Stability

\section{Introduction}

S-systems are proposed in Voit (2000) as a canonical nonlinear model to represent the dynamics of a large class of genetic regulatory networks and metabolic pathways. Altering the production of certain compounds in metabolism is essential for many industrial applications such as cosmetics and food industry. Sometimes, external factors or genetic mutations modify the steady-state of the metabolic pathways. Thus, steering the state of the metabolism back to the desired level is often needed. In this regard, novel intervention

Anirban Mukherjee

anirban@ee.iitkgp.ernet.in

1 Department of Electronics and Communication Engineering, Indian Institute of Information Technology Guwahati, Guwahati, Assam, India

2 Department of Electrical Engineering, Indian Institute of Technology Kharagpur, Kharagpur, West Bengal, India strategies are developed in Meskin et al. (2011), Panja et al. (2013) and the controllability of S-systems is discussed in Radhakrishnan and Voit (2005) based on feedback linearization. At steady-state, the robustness of the nonlinear S-system model is analyzed in Chen et al. (2007), Panja et al. (2013).

The goal of the control strategy is to transfer the dependent variables from an initial steady-state to the desired final ones by manipulating a selected number of independent variables and to improve the robustness by tuning kinetic parameters. It is ensured that the synthetic linearized system remains stable in the Lyapunov sense and will be robust to parameter perturbation. It is ensured that the designed synthetic pathway remains non-negative system. The steady-states of the synthetic system are steered with the minimum control input.

\section{Metabolic Pathways: S-System Model}

Among various models of enzymatic reactions, the S-system model, referring to the synergism and saturation properties of metabolic networks, is as follows:

$\frac{d x_{i}}{d t}=\alpha_{i} \prod_{j=1}^{n+m} x_{j}^{g_{i j}}-\beta_{i} \prod_{j=1}^{n+m} x_{j}^{h_{i j}} i=1,2, \ldots, n$,

The details of parameters of (1) is available in Voit (2000). The synthetic network can be designed by modulating (some of) the dependent and independent variables in the following way:

$\frac{d \bar{x}_{i}}{d t}=r_{i}=\alpha_{i} \prod_{j=1}^{n+m} \bar{x}_{j}^{\tilde{g}_{i j}}-\beta_{i} \prod_{j=1}^{n+m} \bar{x}_{j}^{\tilde{h}_{i j}}, i=1,2, \ldots, n$

where $\tilde{g}_{i j}=g_{i j}+c_{i j}$ and $\tilde{h}_{i j}=h_{i j}+d_{i j}$ are the tuned kinetic variables related to desired dependent variables 
$\bar{x}_{D}=\left[\bar{x}_{i}\right] \in \mathbb{R}^{n}$ for $i=1,2, \ldots, n$. The steady-state vector of $\bar{x}_{D}$ is $x_{D}^{\prime}=\left[x_{i}^{\prime}\right] \in \mathbb{R}^{n}$. The kinetic variables related to tuned independent variables $\quad x_{I}^{\prime}=\left[\bar{x}_{i}\right]=\left[e^{f_{i-n}} x_{i}\right] \in \mathbb{R}^{m} \quad$ for $i=n+1, n+2, \ldots, n+m$, are same as that of the nominal system i.e. $\tilde{g}_{i j}=g_{i j}$ and $\tilde{h}_{i j}=h_{i j}$. The rate of change of concentration of the dependent variable $\bar{x}_{i}$ of the synthetic network is $r_{i}$. The steady-state model of this synthetic Ssystem, (2), is $\left(A_{D}+F\right) Y_{D}^{\prime}=b-A_{I}\left(Y_{I}+f\right)$, where $Y_{D}^{\prime}=$ $\ln \left(x_{D}^{\prime}\right)$ is the steady-state vector of various concentrations in the synthetic network, $f=\left[f_{1} f_{2} \cdots f_{m}\right]^{T} \in \mathbb{R}^{m}$ is the control parameter vector for steering steady-state, and $F=\left[F_{i j}\right]=\left[c_{i j}-d_{i j}\right] \in \mathbb{R}^{n \times n}$ is the control parameter matrix to increase robustness of the metabolic network. The strategy of optimization needs manipulation of some or all of the independent "control" variables, which are typically the enzyme activities, or the magnitudes of transport steps. Additionally, a selected number of elements of $F$ are chosen for manipulation.

\section{Main Results: Analysis of Synthetic Metabolic Network at Steady-State}

\section{Stability Analysis}

The Jacobian matrix (with respect to $\bar{x}_{D}$ ) of the synthetic network, (2), at $\left[x_{D}^{\prime T} x_{I}^{\prime T}\right]^{T}$, is denoted by $J_{D}=\left[\frac{\partial r_{i}}{\partial \bar{x}_{j}}\right]_{x_{D}^{\prime}, x_{I}^{\prime}} \in \mathbb{R}^{n \times n}$ where

$\frac{\partial r_{i}}{\partial \bar{x}_{j}}=\alpha_{i} \frac{\tilde{g}_{i j}}{\bar{x}_{j}} \prod_{k=1}^{n+m} \bar{x}_{k}^{\tilde{g}_{i k}}-\beta_{i} \frac{\tilde{h}_{i j}}{\bar{x}_{j}} \prod_{k=1}^{n+m} \bar{x}_{k}^{\tilde{h}_{i k}} i=1,2, \ldots, n, \bar{x}_{j}>0$

At steady-state, $\left[x_{D}^{\prime T} x_{I}^{\prime T}\right]^{T}$, with $i, j=1,2, \ldots, n$,

$\left.\frac{\partial r_{i}}{\partial \bar{x}_{j}}\right|_{x_{D}^{\prime}, x_{I}^{\prime}}=\frac{\alpha_{i}}{x_{j}^{\prime}}\left(\tilde{g}_{i j}-\tilde{h}_{i j}\right) \prod_{k=1}^{n+m} x_{k}^{\prime \tilde{g}_{i k}}=\frac{\beta_{i}}{x_{j}^{\prime}}\left(\tilde{g}_{i j}-\tilde{h}_{i j}\right) \prod_{k=1}^{n+m} x_{k}^{\prime \tilde{h}_{i k}}$,

The Jacobian matrix, $J_{D}$, can be expressed as $J_{D}=U\left(A_{D}+F\right) V$, where $V=\operatorname{diag}\left\{\frac{1}{x_{1}^{\prime}}, \frac{1}{x_{2}^{\prime}}, \ldots \frac{1}{x_{n}^{\prime}}\right\}>0$ and $U=\operatorname{diag}\left\{\alpha_{1} \prod_{k=1}^{n+m} x_{k}^{\prime} \tilde{g}_{1 k}, \alpha_{2} \prod_{k=1}^{n+m} x_{k}^{\prime \tilde{g}_{2 k}}, \ldots \alpha_{n} \prod_{k=1}^{n+m} x_{k}^{\prime} \tilde{g}_{n k}\right\}>0$. Similarly, the Jacobian matrix, $J_{I} \in \mathbb{R}^{n \times m}$, (with respect to $\left.x_{I}\right)$ can be expressed as $J_{I}=U A_{I} W$, where $W=\operatorname{diag}\left\{\frac{1}{x_{n+1}^{\prime}}, \frac{1}{x_{n+2}^{\prime}}, \ldots \frac{1}{x_{n+m}^{\prime}}\right\}>0$. The Jacobi linearization of the synthetic system (2) can be represented as $\frac{d \Delta x_{D}}{d t}=J_{D} \Delta x_{D}+J_{I} \Delta x_{I}$, where $\Delta x_{D}=\bar{x}_{D}-x_{D}^{\prime}$ and $\Delta x_{I}=\bar{x}_{I}-x_{I}^{\prime}$. Assuming $\Delta x_{I}=0$, the equilibrium of the synthetic system is asymptotically stable (in the Lyapunov sense) if there exists a positive definite matrix $P_{1}=P_{1}^{T}>0$ such that $J_{D}^{T} P_{1}+P_{1} J_{D}<0$. This can be written as
$V^{T}\left(A_{D}+F\right)^{T} U^{T} P_{1}+P_{1} U\left(A_{D}+F\right) V<0$

Defining $U^{T} P_{1} V^{-1}=P$, (3) can be written as

$$
\left(A_{D}+F\right)^{T} P+P^{T}\left(A_{D}+F\right)<0
$$

The equilibrium point considering zero input perturbation $\left(\Delta x_{i}=0\right)$ of the synthetic network is hence Lyapunov stable if there exists a positive definite matrix, $P=P^{T}>0$, such that (4) holds and this is obtained when the eigenvalues of $\left(A_{D}+F\right)$ lie in the open left half of the complex plane.

\section{Analysis of Steady-State Error}

The motivation is to design a control parameter matrix, $F$, and control parameter vector, $f$, which can steer a present steady-state, $Y_{D}$, of a metabolic network (modeled by S-system) to a desired steady-state goal vector, $Y_{D}^{\prime \prime}=\ln \left(x_{D}^{\prime \prime}\right)$. Ideally, this system should reach a desired steady-state goal vector (with zero steady-state error) by designing $F$ and $f . x_{D}^{\prime}=\left[x_{1}^{\prime} x_{2}^{\prime} \cdots x_{n}^{\prime}\right]^{T}$ is the achieved steady-state which may be close (if not same) to the desired steady-state $x_{D}^{\prime \prime}=\left[x_{1}^{\prime \prime} x_{2}^{\prime \prime} \cdots x_{n}^{\prime \prime}\right]^{T}$ of the metabolic network. In contrast to attaining the desired steady-state goal vector, $Y_{D}^{\prime \prime}$, the system may reach a new steadystate, $Y_{D}^{\prime}=\ln \left(x_{D}^{\prime}\right)$, owing to the presence of other metabolic constraints (like the structure of $F$ and $f$ ). Assuming the inversion exists, this can be written as $Y_{D}^{\prime}=\left(A_{D}+F\right)^{-1}\left[b-A_{I}\left(Y_{I}+f\right)\right]$. And the design requirement is to minimize the following upper-bound of the relative steady-state error:

$$
\begin{aligned}
& e_{s s}=\frac{\left\|Y_{D}^{\prime \prime}-Y_{D}^{\prime}\right\|}{\left\|Y_{D}^{\prime \prime}\right\|}=\frac{\left\|Y_{D}^{\prime \prime}-\left(A_{D}+F\right)^{-1}\left[b-A_{I}\left(Y_{I}+f\right)\right]\right\|}{\left\|Y_{D}^{\prime \prime}\right\|} \\
& e_{s s} \leq \frac{\left\|\left(A_{D}+F\right)^{-1}\right\|\left\|\left(E+F Y_{D}^{\prime \prime}+A_{I} f\right)\right\|}{\left\|Y_{D}^{\prime \prime}\right\|}=e_{s s}^{B}
\end{aligned}
$$

where $E=A_{D} Y_{D}^{\prime \prime}-A_{D} Y_{D}$ is known. Now, it is observed that the relative steady-state error bound, $e_{s s}^{B}$, can be minimized by minimizing matrix norm, $\left\|\left(A_{D}+F\right)^{-1}\right\|$, and vector norm, $\left\|\left(E+F Y_{D}^{\prime \prime}+A_{I} f\right)\right\|$.

\section{Perturbation Analysis for Robustness}

The steady-state model of perturbed synthetic network is $\left(A_{D}+F+\Delta A_{D}\right)\left(Y_{D}^{\prime}+\Delta Y_{D}^{\prime}\right)=(b+\Delta b)-\left(A_{I}+\Delta A_{I}\right)\left(Y_{I}+f+\right.$ $\left.\Delta Y_{I}\right)$, where the $\Delta$ terms indicate the perturbation.

Perturbation in the rate constant vector $b$ Let $\Delta Y_{D}^{\prime}$ be the perturbation in the nominal solution when the rate constant vector $b$ is perturbed by $\Delta b$. The perturbations 
$\Delta A_{D}, \Delta A_{I}$ and $\Delta Y_{I}$ are assumed to be zero. The perturbed model of the synthetic network is

$$
\left(A_{D}+F\right)\left(Y_{D}^{\prime}+\Delta Y_{D}^{\prime}\right)=(b+\Delta b)-\left(A_{I}\right)\left(Y_{I}+f\right)
$$

Assuming $\left(A_{D}+F\right)^{-1}$ exists, the perturbed steady-state model (6) is simplified to $\Delta Y_{D}^{\prime}=\left(A_{D}+F\right)^{-1}(\Delta b)$. The rate constants perturbation sensitivity, $S_{b}$, is as follows:

$$
S_{b}=\frac{\left\|\Delta Y_{D}^{\prime}\right\|}{\|\Delta b\|} \leq\left\|\left(A_{D}+F\right)^{-1}\right\|=S_{b}^{B}
$$

Hence, the minimization of right hand side of (7) facilitates to achieve the perturbation rejection ability. Similarly, it is analyzed for $Y_{I}, A_{I}$ and $A_{D}$. Using the inequalities of matrix and vector norms,

$S_{Y_{I}}=\frac{\left\|\Delta Y_{D}^{\prime}\right\|}{\left\|\Delta Y_{I}\right\|} \leq\left\|\left(A_{D}+F\right)^{-1}\right\|\left\|A_{I}\right\|=S_{Y_{I}}^{B}$

$S_{A_{I}}=\frac{\left\|\Delta Y_{D}^{\prime}\right\|}{\left\|\Delta A_{I}\right\|} \leq\left\|\left(A_{D}+F\right)^{-1}\right\|\left\|\left(Y_{I}+f\right)\right\|=S_{A_{I}}^{B}$

$S_{A_{D}}=\frac{\left\|\Delta Y_{D}^{\prime}\right\|}{\left\|\Delta A_{D}\right\|} \leq\left\|\left(A_{D}+F\right)^{-1}\right\|^{2}\left(\|b\|+\left\|A_{I}\right\|\left\|Y_{I}+f\right\|\right)=S_{A_{D}}^{B}$

where, $S_{b}^{B}, S_{Y_{I}}^{B}, S_{A_{I}}^{B}, S_{A_{D}}^{B}$ are the corresponding perturbation sensitivity bounds with respect to $Y_{I}, A_{I}$ and $A_{D}$ respectively.

It is to note that the minimization of $\left\|\left(A_{D}+F\right)^{-1}\right\|$ and $\left\|Y_{I}+f\right\|$ reduces $S_{b}^{B}, S_{Y_{I}}^{B}, S_{A_{I}}^{B}$ and $S_{A_{D}}^{B}$. Thus, the robustness of the synthetic network is achieved.

\section{Synthesis of Robust and Stable Metabolic Network Modeled in S-System Formalism}

Now the principal theorem of this work is proposed. This theorem provides a set of sufficient conditions for the existence of the control parameter matrix, $F$, and control parameter vector, $f$, to achieve steady-state steering with robustness and stability using minimum control effort.

Theorem 1 Let $A_{D} \in \mathbb{R}^{n \times n}$ be non-singular, and $s_{1}, s_{2}, s_{3}$, $s_{4}, s_{5}>0$. A control parameter matrix, $F$, and a control parameter vector, $f$, offer stability and robustness if they are feasible solutions to the following optimization problem:

Minimize $w_{1} s_{1}^{2}+w_{2} s_{2}^{2}+w_{3} s_{3}^{2}+w_{4} s_{4}^{2}+w_{5} s_{5}^{2}$

Subject to

$$
\left[\begin{array}{cc}
\left(A_{D} A_{D}^{T}+F A_{D}^{T}+A_{D} F^{T}\right) & I \\
I & s_{1}^{2} I
\end{array}\right]>0,
$$

$\left[\begin{array}{cc}s_{2}^{2} I & \left(E+F Y_{D}^{\prime \prime}+A_{I} f\right)^{T} \\ \left(E+F Y_{D}^{\prime \prime}+A_{I} f\right) & I\end{array}\right]>0$,

$\left[\begin{array}{cc}s_{3}^{2} I & \left(Y_{I}+f\right)^{T} \\ \left(Y_{I}+f\right) & I\end{array}\right]>0$,

$\left[\begin{array}{cc}s_{4}^{2} I & f^{T} \\ f & I\end{array}\right]>0$

$\left[\begin{array}{cc}s_{5}^{2} I & F^{T} \\ F & I\end{array}\right]>0$,

$\left[\begin{array}{cc}-A_{D}^{T}\left(A_{D}+F\right)-F^{T} A_{D} & \left(A_{D}+P+F\right)^{T} \\ \left(A_{D}+P+F\right) & -I\end{array}\right]<0$,

$P>0$,

$f_{M}>f>f_{m}$,

where $E=A_{D}\left(Y_{D}^{\prime \prime}-Y_{D}\right), f_{m} \in \mathbb{R}^{m}, f_{M} \in \mathbb{R}^{m}$ and $0 \leq w_{i} \leq 1$, $i=1,2, \cdots 5$, are given and satisfy $\sum_{i=1}^{5} w_{i}=1$.

Proof Using Schur complement lemma, (10) can equivalently be written as

$\left(A_{D} A_{D}^{T}+F A_{D}^{T}+A_{D} F^{T}-s_{1}^{-2} I\right)>0$.

It is apparent that if (18) holds, the condition $\left(A_{D} A_{D}^{T}+F A_{D}^{T}+A_{D} F^{T}+F F^{T}-s_{1}^{-2} I\right)>0$ is satisfied. This can equivalently be written as $\left\|\left(A_{D}+F\right)^{-1}\right\|<s_{1}$. By minimizing $s_{1}$ over $F,\left\|\left(A_{D}+F\right)^{-1}\right\|$ is minimized.

Using Schur complement lemma, (11) can equivalently be written as

$s_{2}^{2} I-\left(E+F Y_{D}^{\prime \prime}+A_{I} f\right)^{T}\left(E+F Y_{D}^{\prime \prime}+A_{I} f\right)>0$

Now (19) can equivalently be written as $\left\|\left(E+F Y_{D}^{\prime \prime}+A_{I} f\right)\right\|<s_{2}$. By minimizing $s_{2}$ over $f$ and $F$, $\left\|\left(E+F Y_{D}^{\prime \prime}+A_{I} f\right)\right\|$ is minimized.

Similarly, by using Schur complement lemma, (12) can equivalently be written as $s_{3}^{2} I-\left(Y_{I}+f\right)^{T}\left(Y_{I}+f\right)>0$ which is equivalent to: $\left(Y_{I}+f\right)^{T}\left(Y_{I}+f\right)<s_{3}^{2} I \Leftrightarrow\left\|Y_{I}+f\right\|<s_{3}$. Now, minimizing $s_{3}$ implies the minimization of $\left\|Y_{I}+f\right\|$ that in turn reduces the sensitivity with respect to the perturbations in $A_{I}$ (see (9)) and hence the robustness is improved.

Similarly, it can be shown (13) is for minimization of the control input, $f$, and the control parameter matrix, $F$ is minimized in (14) by minimizing $s_{4}$ and $s_{5}$ respectively. Let $A_{D}^{T} P+P A_{D}+F^{T} P+P F=M$. From (4), the stability implies $M<0$. Now,

$$
\begin{aligned}
& \left(A_{D}+P+F\right)^{T}\left(A_{D}+P+F\right)-M-A_{D}^{T} A_{D} \\
& -A_{D}^{T} F-F^{T} A_{D}=P^{T} P+F^{T} F
\end{aligned}
$$


As $P^{T} P+F^{T} F>0$, from (20), it can be written as

$$
\begin{aligned}
& M<-A_{D}^{T} A_{D}-A_{D}^{T} F-F^{T} A_{D}+ \\
& \left(A_{D}+P+F\right)^{T}\left(A_{D}+P+F\right) \\
& \text { Now, }-\mathrm{A}_{\mathrm{D}}^{\mathrm{T}} \mathrm{A}_{\mathrm{D}}-\mathrm{A}_{\mathrm{D}}^{\mathrm{T}} \mathrm{F}-\mathrm{F}^{\mathrm{T}} \mathrm{A}_{\mathrm{D}} \\
& +\left(A_{D}+P+F\right)^{T}\left(A_{D}+P+F\right)<0,
\end{aligned}
$$

implies $M<0$, i.e., the stability is ensured. Using Schur complement lemma, (22) can be equivalently written as (15). This completes the proof.

\section{S-system Model of AFP in S. cerevisiae}

The S-system model of AFP in Saccharomyces cerevisiae is considered in Voit (2000). The dependent and independent variables are as follows Voit (2000):

Dependent variables: Internal glucose $\left(\mathrm{G}_{I n}\right)-x_{1}$; Glucose-6-phosphate (G6P)- $x_{2}$; $\quad$ Fructose-1,6-diphosphate (FdP)- $x_{3}$; Phophoenolpyruvate (PEP)- $x_{4}$; Adenosine triphosphate (ATP)- $x_{5}$. Independent variables: Glucose uptake $x_{6}=19.7 \mathrm{mM} / \mathrm{min}$; Hexokinase $\mathrm{x}_{7}=68.5 \mathrm{mM} /$ min; Phosphofructokinase (PFK) $x_{8}=31.7 \mathrm{mM} / \mathrm{min}$; Glyceraldehyde-3-phosphate dehydrogenase (GAPD) $x_{9}=49.9$ $\mathrm{mM} / \mathrm{min}$; Pyruvate Kinase (PK) $x_{10}=3440.0 \mathrm{mM} / \mathrm{min}$; Polysaccharide production $x_{11}=14.31 \mathrm{mM} / \mathrm{min}$; Glycerol production $x_{12}=203.0 \mathrm{mM} / \mathrm{min}$; ATPase $x_{13}=25.1 \mathrm{mM} /$ min, $\mathrm{NAD}^{+} / \mathrm{NADH}$ ratio $x_{14}=0.042$.

The rate constant vector, $b$, the transformed independent variable vector, $Y_{I}$, and the transformed dependent variable vector, $Y_{D}$, are computed as $b=[1.259-1.698-3.565$ $1.4583 .559]^{T}, \quad Y_{I}=\ln \left(x_{I}\right)=[2.9814 .2273 .4563 .918 .143$ $2.6615 .3133 .223-3.17]^{T}$, and $Y_{D}=\ln \left(x_{D}\right)=[-3.3660 .014$ $2.217-4.6510 .127]^{T}$ respectively Voit (2000). As a result, the dependent variable vector at steady-state is $x_{D}=e^{Y_{D}}=[0.03451 .01419 .18230 .00961 .1353]^{T}$. The eigenvalues of the system matrix, $A_{D}$ are $-0.6981 \pm 0.6161 i$, $-0.3640 \pm 0.1996 i$, and -0.8222 .

\section{Steady-State Steering and Sensitivity Analysis}

\section{Steady-State Performance}

In order to steer the dependent variables

$x_{D}=[0.03451 .01419 .18230 .00961 .1353]^{T}$ to the desired steady-state goal $x_{D}^{\prime \prime}=\left[x_{1} x_{2} 1.2 x_{3} 1.2 x_{4} x_{5}\right]^{T}$, the optimal solution variables, $s, f, F$, and $P$, are obtained via Theorem 1 and are provided in Table 1 . The structure of $P$ is assumed to be diagonal to ensure the diagonal stability. The eigenvalues of $A_{D}+F$ are $-1.116 \pm 0.8359 i$,
Table 1 Optimal solution variables with weight vector, $w_{t}=\left[\frac{w}{k+2} \frac{k w}{k+2} \frac{w}{k+2} \frac{1-w}{2} \frac{1-w}{2}\right]^{T}, w=0.5, \quad k=5, \quad f_{M}=0.5 *[$ diag $\{1,0,1,1,0,0,0,0,0\}] Y_{I}$ and $f_{m}=-f_{M}$

$\mathbf{s}=\left[\begin{array}{c}1.9156 \\ 0.1299 \\ 12.7127 \\ 1.3651 \\ 0.6319\end{array}\right]$
$\mathbf{f}=\left[\begin{array}{c}-0.6801 \\ 0 \\ -0.7834 \\ -0.878 \\ 0,\end{array}\right]$

$-0.7323 \pm 0.5748 i$, and -0.9038 . As all the stable eigenvalues of $A_{D}+F$ are moved further towards the left compared to that of $A_{D}$, the degree of stability of the synthetic system is improved.

The S-system model of the synthetic AFP (using $f$ and $F$ from the Table 1) is as follows:

$$
\begin{aligned}
\frac{d \bar{x}_{1}}{d t}= & 0.8122 \bar{x}_{2}{ }^{-0.5335} \bar{x}_{6}-2.8632 \bar{x}_{1}^{0.9594} \bar{x}_{5}^{0.1216} \bar{x}_{7}, \\
\frac{d \bar{x}_{2}}{d t}= & 2.8632 \bar{x}_{1}^{0.9656} \bar{x}_{5}^{-0.1019} \bar{x}_{7} \\
& -0.5239 \bar{x}_{2}^{1.1287} \bar{x}_{5}^{-0.394} \bar{x}_{8}^{0.999} \bar{x}_{11}^{0.001}, \\
\frac{d \bar{x}_{3}}{d t}= & 0.5232 \bar{x}_{2}{ }^{0.6679} \bar{x}_{5}^{-0.502} \bar{x}_{8} \\
& -0.0148 \bar{x}_{3}{ }^{0.9803} \bar{x}_{4}^{0.271} \bar{x}_{5}^{0.119} \bar{x}_{9}^{0.944} \bar{x}_{12}^{0.056} \bar{x}_{14}^{-0.575} \\
\frac{d \bar{x}_{4}}{d t}= & 0.022 \bar{x}_{3}{ }^{0.485} \bar{x}_{5}^{-0.1052} \bar{x}_{9} \bar{x}_{14}^{-0.6088} \\
& -0.0945 \bar{x}_{3}{ }^{0.05} \bar{x}_{4}^{0.8154} \bar{x}_{5}^{-0.0822} \bar{x}_{10}, \\
\frac{d \bar{x}_{5}}{d t}= & 0.0913 \bar{x}_{3}{ }^{0.5264} \bar{x}_{4}^{0.1914} \bar{x}_{5}^{0.024} \bar{x}_{9}^{0.5} \bar{x}_{10}^{0.5} \bar{x}_{14}^{-0.304} \\
& -3.2097 \bar{x}_{1}^{0.0158} \bar{x}_{2}^{0.1487} \bar{x}_{5}^{0.7407} \bar{x}_{7}^{0.265} \bar{x}_{8}^{0.265} \bar{x}_{11}^{0.0002} \bar{x}_{13}^{0.47} .
\end{aligned}
$$

where $\quad x_{I}^{\prime}=\left[\bar{x}_{i}\right]=\left[e^{-0.6801} x_{6} x_{7} e^{-0.7834} x_{8} e^{-0.878} x_{9} x_{10} x_{11} x_{12}\right.$ $\left.x_{13} x_{14}\right]^{T}$. This model (23) is devised to become non-negative, which needs to be satisfied for synthetic system owing to non-negative physical constraints Bernstein and Bhatt (Bernstein and Bhatt).

The value of $w_{t}$ is chosen (as given in Table 1) to provide robustness and control effort along-with the stringent criterion on steady-state error. The biochemical map of unperturbed synthetic AFP are shown in Fig. 1. For a desired steady-state goal of $x_{D}^{\prime \prime}=\left[x_{1} x_{2} 1.2 x_{3} 1.2 x_{4} x_{5}\right]^{T}=$ $\left[\begin{array}{ll}0.03451 .014111 .0188 & 0.01151 .1353\end{array}\right]^{T}$, the dependent variables attain the steady-state $x_{D}^{\prime}=[0.03451 .0461$ 11.23160.01221.1901] $]^{T}$.

The perturbation sensitivity bounds (normalized by the respective maximum values) and the relative steady-state 


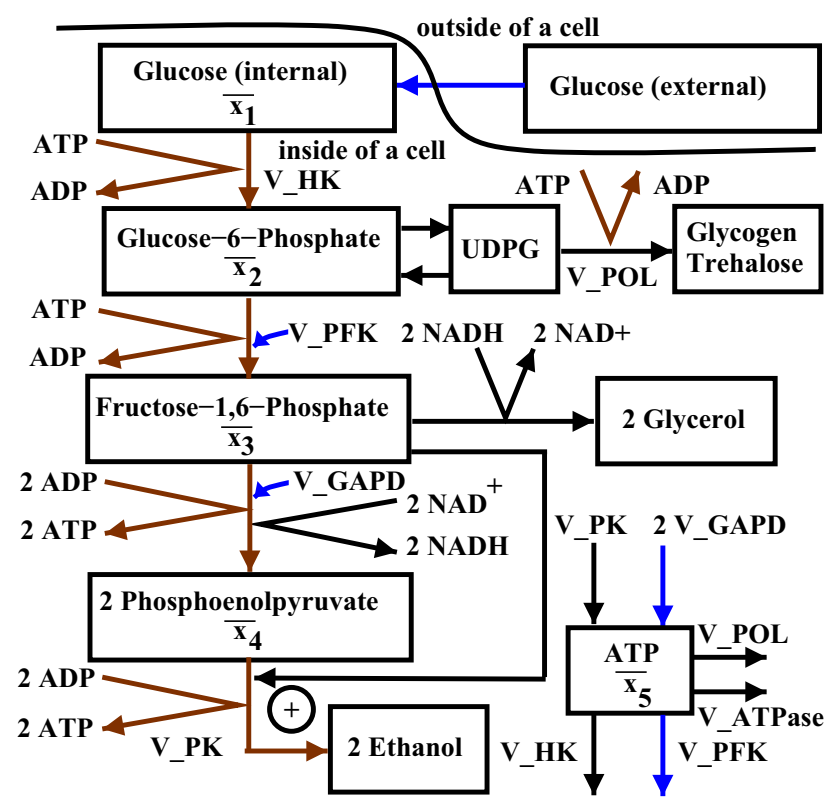

Fig. 1 Biochemical map of the synthetic AFP described by (23). Blue and brown linesshow the effect of $f$ and $F$ respectively (color figure online)

error bound, $e_{s s}^{B}$, are shown in Fig. $2 \mathrm{a}, \mathrm{b}$ respectively. Similarly, the variation in control parameter effort and control input effort (both normalized) are shown in Fig. 3a, $\mathrm{b}$, respectively. A biochemical engineer can choose the value of $w$ based on the trade-off among robustness, steady-state error and control effort $(\|F\|$ and $\|f\|)$.

\section{Biochemical Significance and Implementation}

The biochemical rationale of the synthesis of the network depends on controlling the catalytic efficiency of the enzyme, substrate availability, and concentration of dependent variables $\left(X_{1}, \ldots, X_{5}\right)$ and independent variables $\left(X_{6}, X_{8}\right.$ and $\left.X_{9}\right)$ via stability and robustness. The metabolism of the cell relies on modulation of expression of a gene that codes for an enzyme by recombinant DNA technology. For change in protein expression the use of synthetic promoter, translation modulators such as internal ribosomal entry sites, upstream open reading frames and optimized RNA secondary structure have been practised Klein-Marcuschamer et al. (2010). The intervention targets are selected based on the kinetic importance of the reaction step.

\section{Conclusion}

A robust design technique is proposed in an LMI framework to steer steady-states of metabolic networks, modeled in S-system. The stability issue, in the Lyapunov sense, and the steady-state error bound are considered in synthesizing the network. The parameter sensitivity bounds are minimized for improved robustness. As a future scope, the present design framework can be extended by involving the concept of positive systems, the diagonal stability and the input-to-state stability for non-zero input perturbation.
Fig. 2 a $S_{A_{D}}^{B}$ versus $w, \mathbf{b} e_{s s}^{B}$, versus $w$

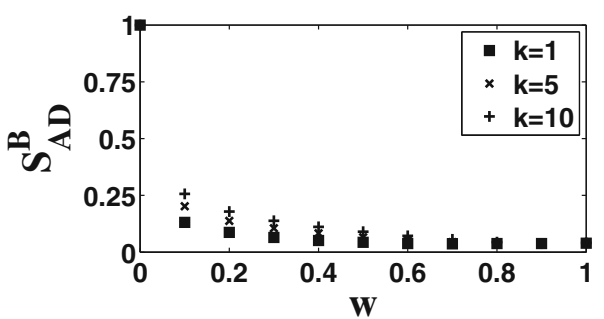

(a)

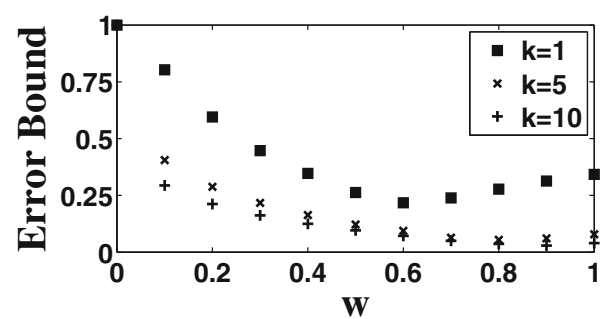

(b)
Fig. 3 a $\|F\|$ versus $w$. b $\|f\|$ versus $w$

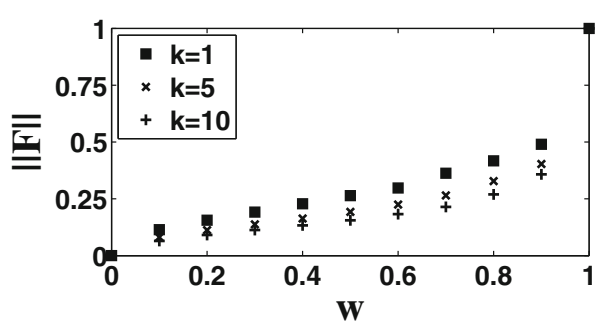

(a)

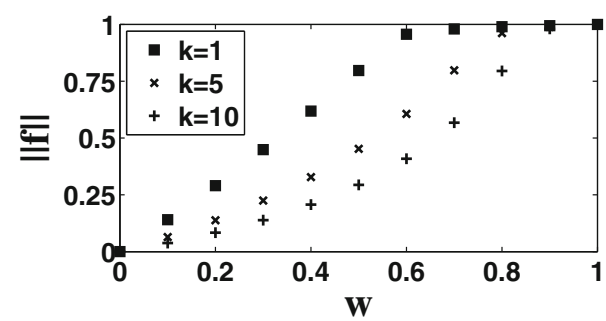

(b) 


\section{References}

Bernstein, DS, Bhatt SP: Nonnegativity, reducibilty, and semistability of mass action kinetics. In: IEEE Conference on Decision and Control (CDC'99)

Chen BS, Wu WS, Wang YC, Li WH (2007) On the robust circuit design schemes of biochemical networks: steady-state approach. IEEE Trans Biomed Circuits Syst 1(2):91-104

Klein-Marcuschamer D, Yadav VG, Ghaderi A, Stephanopoulos GN (2010) De novo metabolic engineering and the promise of synthetic DNA. Adv Biochem Eng Biotechnol 120:101-131

Meskin N, Nounou HN, Nounou M, Datta A, Dougherty ER (2011) Intervention in biological phenomena modeled by S-systems. IEEE Trans Biomed Eng 58(5):1260-1267
Panja S, Patra S, Mukherjee A, Basu M, Sengupta S, Dutta PK (2013) An optimization-based design framework for steering steady states and improving robustness of glycolysis-glycogenolysis pathway. IEEE Trans Biomed Eng 60(2):554-561

Panja S, Patra S, Mukherjee A, Basu M, Sengupta S, Dutta PK (2013) Robustness of TCA cycle at steady-state: an LMI-based analysis and synthesis framework. IEEE Trans. Nanobioscience 12(2):128-134

Radhakrishnan EA, Voit EO (2005) Controllability of non-linear biochemical systems. Math Biosci 196(1):99-123

Voit EO (2000) Computational Analysis of Biochemical Systems: A Practical Guide for Biochemists and Molecular Biologists. Cambridge University Press, Cambridge 